\title{
CXCR4-Targeted PET Imaging of Central Nervous System B-Cell Lymphoma
}

Peter Herhaus ${ }^{1}$, Jana Lipkova ${ }^{2}$, Felicitas Lammer ${ }^{3}$, Igor Yakushev ${ }^{4}$, Tibor Vag ${ }^{4}$, Julia Slotta-Huspenina ${ }^{5}$, Stefan Habringer ${ }^{3,6}$, Constantin Lapa ${ }^{7,8}$, Tobias Pukrop ${ }^{9}$, Dirk Hellwig ${ }^{10}$, Benedikt Wiestler ${ }^{2}$, Andreas K. Buck ${ }^{7}$, Martina Deckert ${ }^{11}$, Hans-Jürgen Wester ${ }^{12}$, Florian Bassermann ${ }^{1}$, Markus Schwaiger ${ }^{4}$, Wolfgang Weber ${ }^{2}$, Björn Menze ${ }^{13}$, and Ulrich Keller ${ }^{1,3,14,15}$

\begin{abstract}
${ }^{I}$ Internal Medicine III, School of Medicine, Technische Universität München, Munich, Germany; ${ }^{2}$ Department of Neuroradiology, School of Medicine, Technische Universität München, Munich, Germany; ${ }^{3}$ Department of Hematology, Oncology, and Tumor Immunology (Campus Benjamin Franklin), Charité-Universitätsmedizin Berlin, Berlin, Germany; ${ }^{4}$ Department of Nuclear Medicine, School of Medicine, Technische Universität München, Munich, Germany; ${ }^{5}$ Institute of Pathology, Technische Universität München, Munich, Germany; ${ }^{6}$ Berlin Institute of Health (BIH), Berlin, Germany; ${ }^{7}$ Department of Nuclear Medicine, Universitätsklinikum Würzburg, Würzburg, Germany; ${ }^{8}$ Department of Nuclear Medicine, University Hospital Augsburg, Augsburg, Germany; 9 Internal Medicine III, Universitätsklinikum Regensburg, Regensburg, Germany; ${ }^{10}$ Department of Nuclear Medicine, Universitätsklinikum Regensburg, Regensburg, Germany; ${ }^{11}$ Insitute of Neuropathology, Faculty of Medicine, University of Cologne, and University Hospital Cologne, Cologne, Germany; ${ }^{12}$ Institute of Pharmaceutical Radiochemistry, Technische Universität München, Munich, Germany; ${ }^{13}$ Informatics Department, Technische Universität München, Munich, Germany; ${ }^{14}$ Max Delbrück Center for Molecular Medicine, Berlin, Germany; and ${ }^{15}$ German Cancer Research Center and German Cancer Consortium, Heidelberg, Germany
\end{abstract}

C-X-C chemokine receptor 4 (CXCR4) is a transmembrane chemokine receptor involved in growth, survival, and dissemination of cancer, including aggressive B-cell lymphoma. MRI is the standard imaging technology for central nervous system (CNS) involvement of B-cell lymphoma and provides high sensitivity but moderate specificity. Therefore, novel molecular and functional imaging strategies are urgently required. Methods: In this proof-of-concept study, 11 patients with lymphoma of the CNS (8 primary and 3 secondary involvement) were imaged with the CXCR4-directed PET tracer ${ }^{68} \mathrm{Ga}$-pentixafor. To evaluate the predictive value of this imaging modality, treatment response, as determined by MRI, was correlated with quantification of CXCR4 expression by ${ }^{68} \mathrm{Ga}$-pentixafor PET in vivo before initiation of treatment in 7 of 11 patients. Results: ${ }^{68} \mathrm{Ga}$-pentixafor PET showed excellent contrast with the surrounding brain parenchyma in all patients with active disease. Furthermore, initial CXCR4 uptake determined by PET correlated with subsequent treatment response as assessed by MRI. Conclusion: ${ }^{68} \mathrm{Ga}$-pentixafor PET represents a novel diagnostic tool for CNS lymphoma with potential implications for theranostic approaches as well as response and risk assessment.

Key Words: central nervous system lymphoma; CXCR4; in vivo imaging; PET; MRI

J Nucl Med 2020; 61:1765-1771

DOI: 10.2967/jnumed.120.241703

Received Jan. 5, 2020; revision accepted Mar. 19, 2020.

For correspondence or reprints contact: Ulrich Keller, Department of Hematology, Oncology, and Tumor Immunology (Campus Benjamin Franklin), Charité-Universitätsmedizin Berlin, Hindenburgdamm 30, 12203 Berlin, Germany.

E-mail: ulrich.keller@charite.de

Published online Apr. 24, 2020.

COPYRIGHT @ 2020 by the Society of Nuclear Medicine and Molecular Imaging.
$\mathbf{L}$ ymphoma of the central nervous system (CNSL) is a rare malignancy and arises as a primary or secondary form. Primary CNSL is confined to the central nervous system (CNS) and constitutes $2 \%-4 \%$ of all primary brain tumors (1-5). Secondary CNSL appears as simultaneous involvement of the CNS next to systemic (peripheral) lymphoma at first diagnosis or as CNS relapse (6-8). Primary CNSL is defined as diffuse large B-cell lymphoma confined to the CNS. In addition, systemic diffuse large B-cell lymphoma may also involve the CNS as secondary lymphoma (6,9-11).

Currently, the standard imaging modality in patients with suspected CNSL is contrast-enhanced cranial MRI. Although diffusionweighted imaging can be helpful in discriminating CNSL from other brain tumors, such as glioblastoma, it offers only moderate specificity (12). Moreover, MRI frequently bears uncertainty in defining a complete remission (13). PET with ${ }^{18} \mathrm{~F}-\mathrm{FDG}$ is not yet sufficiently evaluated as diagnostic imaging in this disease and bears limitations due to the high physiologic glucose uptake in healthy brain parenchyma (14). Diagnosis has to be histopathologically confirmed, preferably by stereotactic biopsy from a CNS lesion, which may be a challenge because of the localization of CNSL in poorly accessible or difficult-to-biopsy brain regions (15). Thus, identification and implementation of targeted imaging modalities are highly desirable to facilitate diagnostic workup in CNSL at initial onset and for restaging.

First-line treatment for CNSL is systemic chemotherapy consisting of drugs that pass the blood-brain barrier. High-dose methotrexate as the most effective single agent represents the backbone of treatment (16). Combination chemotherapies with methotrexate and other agents that cross the blood-brain barrier, such as highdose cytarabine, thiotepa, or ifosfamide, show increased overall response rates, with further improvement by adding the CD20directed antibody rituximab (17-19). Consolidation therapy with high-dose chemotherapy and autologous stem cell transplantation 
has further improved overall survival but is restricted, because of tolerability issues, to younger patients in good condition (8). For patients relapsing after first-line treatment, there is no established salvage therapy; however, repeated methotrexate-based therapies can again provide remissions. Whole-brain radiotherapy often remains the only suitable treatment option for relapsed CNSL but is associated with neurotoxicity that is often severe enough to compromise life or quality of life (20-22).

C-X-C chemokine receptor 4 (CXCR4), with its sole known ligand CXCL12, plays an important role during embryonic organogenesis and orchestrates important immunologic functions as a master regulator of leukocyte migration, leukocyte homing, and hematopoietic stem and progenitor cell retention in bone marrow niches (23-28). Malignant cells often hijack the physiologic functions of the CXCL12/CXCR4 axis, and consequently, high CXCR4 expression has been associated with an adverse prognosis in various malignancies. The tumor cells of primary CNSL are characterized by their strong and consistent expression of CXCR4 (29-31).

CXCR4-directed PET imaging with the tracer ${ }^{68} \mathrm{Ga}$-pentixafor has been proven a suitable in vivo imaging modality for CXCR4 expression in various malignancies (32-37). Furthermore, CXCR4directed endoradiotherapy with pentixather, the therapeutic twin of the imaging peptide pentixafor, was performed on small patient cohorts with multiple myeloma, acute myeloid leukemia, and diffuse large B-cell lymphoma (38-40). Here, we present data from a proof-of-concept study investigating the potential of CXCR4directed PET imaging with ${ }^{68} \mathrm{Ga}$-pentixafor in patients with CNSL.

\section{MATERIALS AND METHODS}

\section{Patients}

The characteristics of the PET imaging patient cohort are detailed in Table 1. All patients had histologically confirmed B-cell lymphoma. As previously reported for other ${ }^{68} \mathrm{Ga}$-labeled peptides $(41),{ }^{68} \mathrm{Ga}$ pentixafor was administered according to the German Medicinal Products Act, AMG $\S 132 \mathrm{~b}$, and in accordance with the responsible regulatory agencies (Regierung von Oberbayern, Regierung von Oberfranken, and

TABLE 1

Patient Characteristics

\begin{tabular}{cccccc}
\hline Patient & Sex & Age $(\mathrm{y})$ & Diagnosis & $\begin{array}{c}\text { Disease } \\
\text { stage }\end{array}$ & $\begin{array}{c}\text { No. of } \\
\text { relapses }\end{array}$ \\
\hline 1 & M & 50 & PCNSL & First diagnosis & 0 \\
\hline 2 & M & 67 & PCNSL & First diagnosis & 0 \\
3 & F & 63 & DLBCL & CNS relapse & 3 \\
\hline 4 & F & 55 & DLBCL & CNS relapse & 1 \\
5 & F & 65 & PCNSL & First diagnosis & 0 \\
6 & M & 80 & PCNSL & First diagnosis & 0 \\
7 & M & 59 & PCNSL & First diagnosis & 0 \\
\hline 8 & M & 66 & PCNSL & First diagnosis & 0 \\
9 & M & 58 & PCNSL & First diagnosis & 0 \\
10 & M & 64 & PCNSL & First diagnosis & 0 \\
11 & M & 79 & DLBCL & CNS relapse & 1 \\
\hline
\end{tabular}

$\mathrm{PCNSL}=$ primary CNSL; DLBCL $=$ diffuse large B-cell lymphoma.
Regierung von Unterfranken). All patients gave written informed consent before the investigations. The responsible ethics committees approved data analysis.

This study was an observational pilot study used to conduct explorative analyses. As it was not a confirmatory study, there were no prespecified hypotheses that would have allowed for sample size calculation. Therefore, the sample size was chosen to serve this purpose. It enabled the computation of descriptive and explorative statistics.

\section{Synthesis of ${ }^{68} \mathrm{Ga}$-Pentixafor}

${ }^{68} \mathrm{Ga}$-pentixafor was synthesized in a fully automated, goodmanufacturing-practice-compliant procedure using a GRP module (Scintomics $\mathrm{GmbH}$ ) equipped with disposable single-use cassette kits $(\mathrm{ABX})$, applying a method $(32,33)$ and standardized labeling sequence previously described (42). Before injection, the quality of ${ }^{68} \mathrm{Ga}$-pentixafor was assessed according to the standards described in the European Pharmacopoeia for ${ }^{68} \mathrm{Ga}$ edotreotide (monograph 01/2013: 2482).

\section{PET/CT and PET/MRI Studies}

Four of $11{ }^{68} \mathrm{Ga}$-pentixafor scans were performed on dedicated PET/CT scanners (Biograph mCT 64 or mCT 40; Siemens Medical Solutions), and 7 of $11{ }^{68} \mathrm{Ga}$-pentixafor scans were performed on a PET/MRI device (Biograph mMR; Siemens Medical Solutions). Before undergoing ${ }^{68} \mathrm{Ga}$-pentixafor scanning, the patients fasted at least $4 \mathrm{~h}$. Injected activities ranged from 83 to $275 \mathrm{MBq}(1.0-2.9 \mathrm{MBq} / \mathrm{kg})$. The range of injected activities was $83-275 \mathrm{MBq}(1.0-2.8 \mathrm{MBq} / \mathrm{kg})$ for PET/CT and 96-217 MBq (1.0-2.9 MBq/kg) for PET/MRI. Corresponding $\mathrm{CT}$ scans for attenuation correction were acquired using a low-dose protocol $(20 \mathrm{mAs}, 120 \mathrm{keV}, 512 \times 512$ matrix, 5 -mm slice thickness, increment of $30 \mathrm{~mm} / \mathrm{s}$, rotation time of $0.5 \mathrm{~s}$, and pitch index of 0.8 ) including the base of the skull to the proximal thighs. In PET/MRI, a coronal 2-point Dixon 3-dimensional volumetric interpolated examination T1-weighted MRI sequence was first performed for generation of attenuation maps as previously published (43). In addition, both a coronal T1-weighted turbo spin-echo sequence (repetition time/echo time, $600 \mathrm{~ms} / 8.7 \mathrm{~ms}$; slice thickness, $5 \mathrm{~mm}$; matrix, $384 \times 230$ ) and a T2-weighted short- $\tau$ inversion recovery sequence with fat suppression (repetition time/echo time/inversion time, $5,000 \mathrm{~ms} / 56 \mathrm{~ms} / 220 \mathrm{~ms}$; slice thickness, $5 \mathrm{~mm}$; matrix, $106 \times 256)$ were acquired. Consecutively, PET emission data were acquired in 3-dimensional mode with a $200 \times 200$ matrix and a 2 to 3-min emission time per bed position. After decay and scatter correction, PET data were reconstructed iteratively with attenuation correction using dedicated software (Siemens Esoft).

\section{PET, CT, and MR Analysis}

All CT and MRI scans were scored by a board-certified radiologist, and all PET scans were scored by a board-certified nuclear medicine physician. All PET scans were interpreted in a binary visual fashion as positive for disease or negative for disease according to the criteria previously described (44). Lesions were defined as PET-positive if both imaging specialist investigators were able to unambiguously visually identify the lesions on the PET images. Semiquantitative analysis comprised calculation of $\mathrm{SUV}_{\max }$ as well as $\mathrm{SUV}_{\text {mean }}$ by 2-dimensional regions of interest with a diameter of $1.5 \mathrm{~cm}$ around the hottest pixel. If there was more than one CNS lesion, the biggest lesion was assessed for analysis. For the calculation of tumor-to-background ratio, background was measured within healthy parenchyma on the contralateral brain region.

\section{Immunohistochemistry of Patient Biopsy Material}

Immunohistochemistry for CXCR4 expression was conducted as follows: after heat-induced antigen retrieval $(10 \mathrm{mM}$ citrate buffer, 
pH 6, $20 \mathrm{~min}$ ) and protein and peroxidase blocking (universal block [Dako] and 3\% normal goat serum [Abcam]), immunohistochemistry was performed with a Dako autostainer using an antibody against CXCR4 (1:30 [Abcam], ab124824, clone UMB-2). Antibody was detected using the Dako EnVision horseradish peroxidase rabbit labeled polymer visualized by diaminobenzidine (KPL). Counterstaining was performed with hematoxylin. Immunohistochemistry for CD20 was performed using an automated immunostainer with the iVIEW diaminobenzidine detection kit (Ventana Medical Systems, Roche) according to the company's protocols for open procedures. CD20 antibody was used according to the manufacturer's instructions. Appropriate positive controls were used to confirm the adequacy of the staining.

\section{Computed Analysis of Prognostic Value of CXCR4-Directed PET Imaging}

T1-weighted contrast-enhanced (T1c) MRI scans, fluid attenuation inversion recovery (FLAIR) MRI scans, and CXCR4-directed PET scans were acquired before lymphoma treatment. After treatment, patients were monitored with T1c and FLAIR follow-up scans. All scans were registered to the pretreatment T1c MRI scan. PET scans were normalized by subtracting a mean PET uptake value from a healthy-appearing region within the brain. Despite the diagnostic

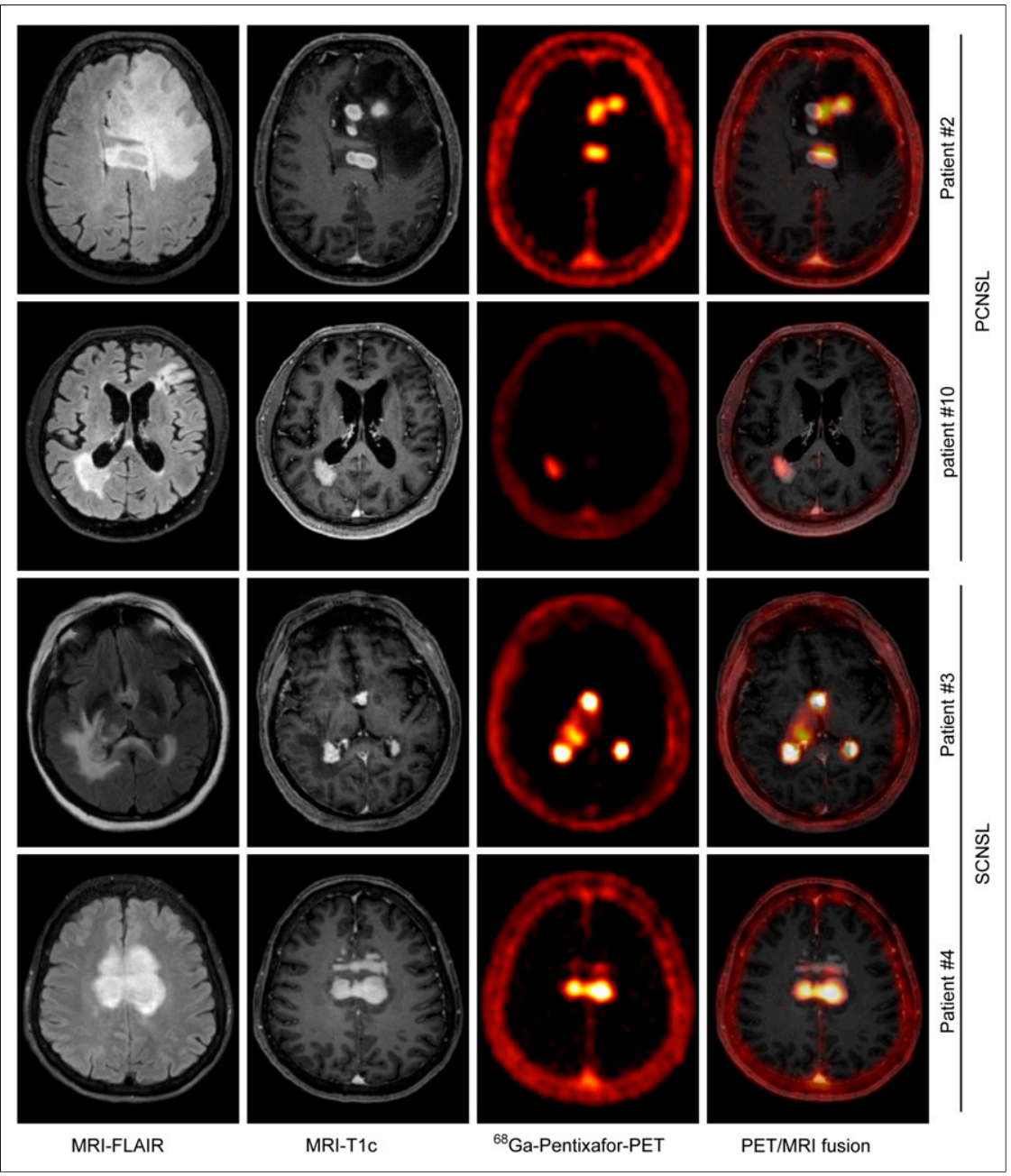

FIGURE 1. Correlation of CXCR4-directed PET with MRI-determined lymphoma lesions. Depicted are representative MR images (T1C and FLAIR sequences) and corresponding CXCR4-directed PET images and fusion of MRI (FLAIR) and PET for 2 patients with primary CNSL (PCNSL) and secondary CNSL (SCNSL), respectively. importance of the FLAIR scan, it was not included in the prognostic performance study. The reason was that the edema surrounding the lesion might be difficult to distinguish from a tissue response to the treatment.

To evaluate the prognostic value of CXCR4-directed PET imaging, the relation between the treatment efficiency and various tumor properties extracted from the pretreatment T1c MRI and PET scans was computed. To account for the variability across lesions and patients, the analysis was performed in both a patientwise and a lesionwise manner. Only patients with available follow-up scans were considered, resulting in 7 patients with a total of 14 individual lesions.

The efficacy of the treatment, $\eta$, was defined as a relative change in tumor volume detected by T1c MRI from the first and the last available scans, that is,

$$
\eta=\frac{\mathrm{V}^{\text {first }}(\mathrm{T} 1 \mathrm{c})-\mathrm{V}^{\text {last }}(\mathrm{T} 1 \mathrm{c})}{\mathrm{V}^{\text {first }}(\mathrm{T} 1 \mathrm{c})} \times 100 \% .
$$

Eq. 1

To account for the treatment response, the following properties were computed for each lesion from the pretreatment scans: volume of the T1c lesion, $\mathrm{V}(\mathrm{T} 1 \mathrm{c})$, in MRI; volume of the PET-enhanced lesion, $\mathrm{V}(\mathrm{PET})$; maximum PET uptake value inside each lesion, $\max (\mathrm{PET})$; and PET uptake values integrated across the PET-enhancing lesion, $\int(\mathrm{PET})$. Since PET scans were normalized as mentioned previously, the term $\mathrm{SUV}_{\max }$ is not appropriate in this setting.

More specifically, the V(T1c) was computed as the volume of all voxels within the T1c-enhancing lesion:

$$
\mathrm{V}(\mathrm{T} 1 \mathrm{c})=\sum_{i \in I} \operatorname{sT} 1 \mathrm{c}(i) d v
$$

Eq. 2

where $\mathrm{sT} 1 \mathrm{c}(i)$ is a binary segmentation of the T1c-enhancing tumor segmented by a boardcertified radiologist. The parameter $i$ denotes an index across all voxels of the input image I, and $d v$ denotes the volume of each voxel.

$\mathrm{V}(\mathrm{PET})$ is defined in a similar way:

$$
\mathrm{V}(\mathrm{PET})=\sum_{i \in \Omega} \operatorname{sPET}(i) d v, \quad \text { Eq. } 3
$$

where $\operatorname{sPET}(i)$ is a binary segmentation of the PET-enhancing lesion $\Omega$. The region $\Omega$ is defined as a region of the PET enhancement obtained after subtracting the $\mathrm{SUV}_{\text {mean }}$ measured within healthy parenchyma on the contralateral brain region of each patient.

Finally, the integrated PET uptake values are defined as:

$$
\int(\mathrm{PET})=\int_{i \in \Omega} \phi(i) d v \approx \sum_{i \in \Omega} \phi(i) d v,
$$

where $\phi(i)$ denotes the PET uptake value at voxel $i$ inside the regions of the PET-enhancing lesion $\Omega$ and $d v$ is volume of the voxel $i$.

The matrices $\mathrm{V}(\mathrm{T} 1 \mathrm{c})$ and $\mathrm{V}(\mathrm{PET})$ both measure tumor burden but do not account for the tumor activity. The max(PET) describes 
TABLE 2

SUV $_{\text {max }}$, SUV $_{\text {mean, }}$ and Corresponding Tumor-to-Background Ratio

\begin{tabular}{|c|c|c|c|c|c|c|}
\hline \multirow[b]{2}{*}{ Patient } & \multicolumn{3}{|c|}{$\mathrm{SUV}_{\max }$} & \multicolumn{3}{|c|}{$\mathrm{SUV}_{\text {mean }}$} \\
\hline & Tumor & Background & Ratio & Tumor & Background & Ratio \\
\hline 1 & No lesion & No lesion & No lesion & No lesion & No lesion & No lesion \\
\hline 2 & 4.2 & 0.2 & 18.3 & 2.8 & 0.2 & 17.3 \\
\hline 3 & 23.3 & 0.6 & 38.8 & 16.1 & 0.5 & 32.2 \\
\hline 4 & 5.5 & 0.3 & 16.2 & 3.2 & 0.2 & 16.2 \\
\hline 5 & 7.0 & 0.4 & 18.4 & 4.5 & 0.2 & 19.6 \\
\hline 6 & 4.7 & 0.4 & 13.4 & 3.1 & 0.2 & 18.2 \\
\hline 7 & 6.0 & 0.1 & 75.1 & 4.0 & 0.01 & 201.0 \\
\hline 8 & 10.1 & 0.8 & 13.2 & 5.6 & 0.2 & 30.9 \\
\hline 9 & 8.3 & 0.1 & 83.0 & 5.4 & 0.1 & 54.0 \\
\hline 10 & 4.6 & 0.2 & 23.0 & 3.2 & 0.2 & 15.9 \\
\hline 11 & 5.4 & 0.3 & 21.6 & 3.4 & 0.2 & 21.2 \\
\hline
\end{tabular}

the tumor activity, but only from a single voxel, neglecting the tumor burden. $\int(\mathrm{PET})$ reflects both the tumor activity and the tumor burden.

In the patient-based study, the same measures were computed across all lesions of each patient.

For each of the extracted tumor properties, the Pearson correlation with the treatment efficiency was computed together with the $P$ value describing the significance of the correlation.

\section{RESULTS}

To address the feasibility of CXCR4-directed PET imaging in patients with CNSL, this proof-of-concept study evaluated 11 patients with the PET tracer ${ }^{68} \mathrm{Ga}$-pentixafor. PET imaging was combined either with MRI (in 7 patients) or with CT (in 4 patients; no local hybrid PET/MRI available). All patients underwent MRI as the standard imaging modality in CNSL during diagnostic workup at CNSL diagnosis. All 8 patients with primary CNSL were investigated at the time of initial manifestation, whereas the 3 patients with secondary CNSL all had a CNS relapse of diffuse large B-cell lymphoma at the time point of analysis. Of note, 8 of 11 patients had received corticosteroids before CXCR4directed PET imaging. The patient characteristics are detailed in Table 1.

\section{Suitability of ${ }^{68} \mathrm{Ga}$-Pentixafor PET Imaging for Detection of CNSL}

CXCR4-directed PET imaging with ${ }^{68} \mathrm{Ga}$-pentixafor was visually positive in 10 of the 11 studied patients. The lymphoma lesions determined by MRI T1c sequences displayed high CXCR4 tracer uptake in ${ }^{68} \mathrm{Ga}$-pentixafor PET imaging. In contrast, the healthy brain parenchyma and the surrounding brain edema seen in the MRI FLAIR sequences were always evaluated as CXCR4directed PET-negative (Fig. 1). Semiquantitative analysis revealed a ${ }^{68}$ Ga-pentixafor-derived PET SUV max $_{\text {of }}$ 4.2-23.3 within the lymphoma lesions with a high tumor-to-background ratio ranging from 13.2 to 83.0 (Table 2 shows all individual patient values).

The only patient who showed no tracer uptake as assessed by CXCR4-directed PET imaging had undergone extensive neurosurgical biopsy of the entire lymphoma lesion and 4 wk of corticosteroid treatment before imaging. PET negativity in this patient was underscored by the lack of lymphoma lesions in the corresponding MR images. Therefore, it can be reasonably assumed that the patient presented without active disease at the time of ${ }^{68} \mathrm{Ga}$-pentixafor PET imaging (Supplemental Fig. 1; supplemental materials are available at http://jnm. snmjournals.org).

CXCR4 expression in vivo as determined by CXCR4-directed PET was validated by positive immunohistochemistry for CXCR4. In 9 of 11 patients, for whom there were lymphoma biopsy samples that had been 
obtained near the time of the PET imaging, CXCR4 was highly expressed within the lymphoma lesions (Fig. 2).

\section{CXCR4-Directed PET Imaging as a Biomarker to Predict Treatment Response}

To further evaluate the potential clinical relevance beyond lymphoma imaging, and in particular, to investigate a correlation between CXCR4 tracer uptake and therapy response, a computed analysis was performed for all 7 patients for whom posttreatment MRI scans were available. Response to treatment, calculated as $\eta$ from the sequential T1c MRI sequences, was compared with 4 properties from the respective pretreatment MRI and CXCR4directed PET scans (details on treatment and treatment response are provided in Supplemental Figs. 2 and 3). The 4 analyzed properties were $\mathrm{V}(\mathrm{T} 1 \mathrm{c}), \mathrm{V}(\mathrm{PET}), \max (\mathrm{PET})$, and $\int(\mathrm{PET})$. This analysis revealed that neither in the lesion-based nor in the patient-based analysis did the tumor volume determined by MRI or by CXCR4directed PET imaging (V(T1c), ranging from 0.69 to $20.08\left[\mathrm{~cm}^{3}\right]$, and $\mathrm{V}(\mathrm{PET})$, ranging from 0.43 to $\left.8.88\left[\mathrm{~cm}^{3}\right]\right)$ represent a predictive marker for treatment response (Figs. 3A, 3B, and 3E).

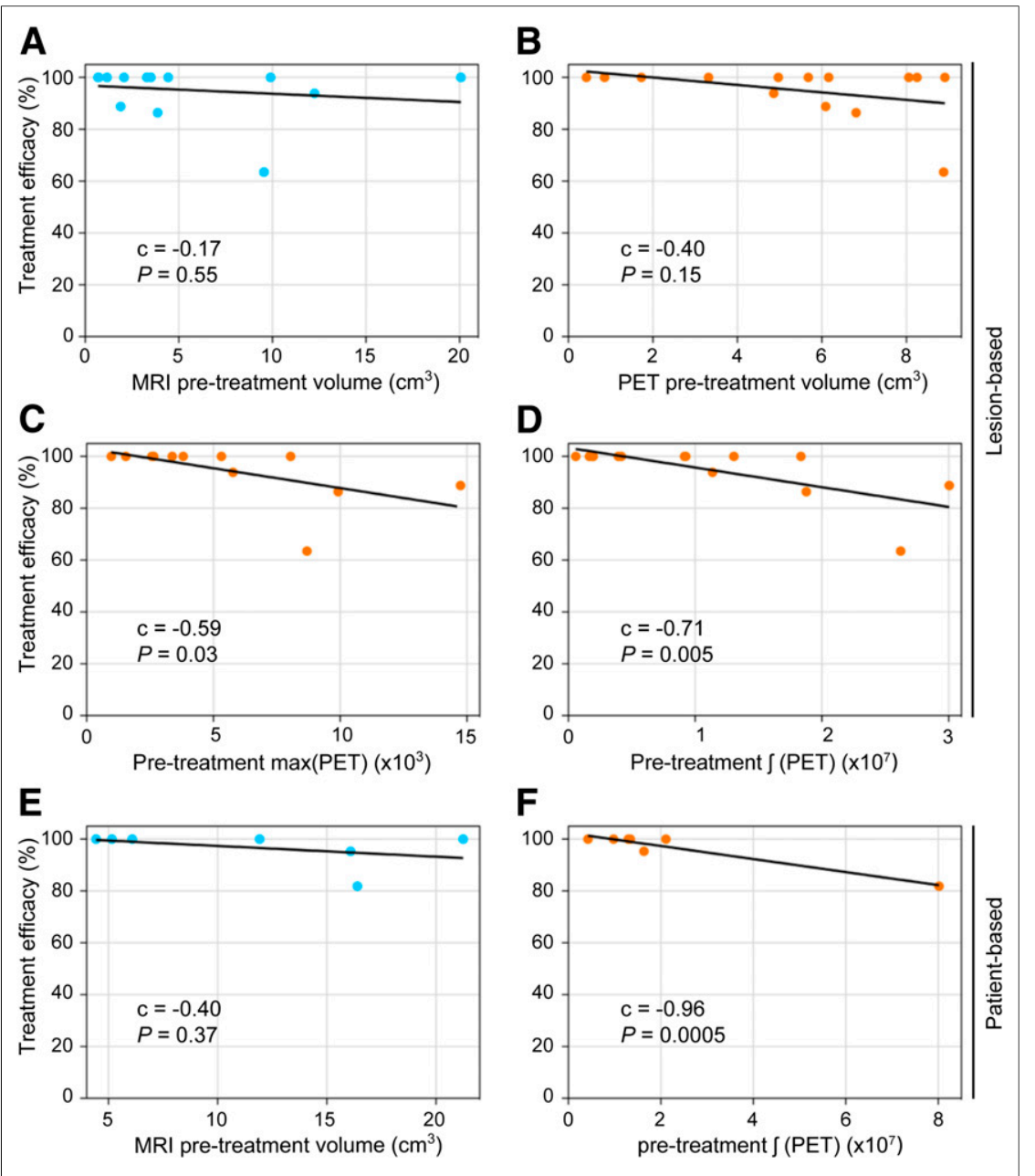

FIGURE 3. CXCR4-directed PET prediction of treatment response. Depicted are correlations between $\eta$ and properties extracted from pretreatment scans: V(T1c) in MRI ( $A$ and $E), V(P E T)(B)$, $\max (P E T)(C)$, and $\int(P E T)(D$ and $F)$. A-D depict results from lesion-based approach. Each dot represents 1 of 14 diagnosed lymphoma lesions. In E and F, results from patient-based approach are shown. Each dot represents results from 1 of 7 patients. Pearson correlation coefficient (c) and $P$ value are reported inside each plot. Least-squares regression line is shown in black.
CXCR4-directed PET uptake values given by $\max (\mathrm{PET})$ and $\int(\mathrm{PET})$, however, had a significant prognostic value both in the lesion-based and in the patient-based analysis (Figs. 3C, 3D, and $3 F$ ). These results imply that lymphoma lesions with lower CXCR4 tracer uptake as assessed by CXCR4-PET were associated with a better response to standard methotrexate-based treatment. Specifically, $\int(\mathrm{PET})$ was the most significant predictive factor in the present study.

\section{DISCUSSION}

Clinical challenges in CNSL involve diagnostic work-up, risk stratification, and optimization of treatment. This proof-of-concept study identified CXCR4-directed PET imaging with ${ }^{68} \mathrm{Ga}$-pentixafor as a novel imaging modality for CNSL with putative additional value when performed in combination with standard MRI. CXCR4directed PET imaging provides excellent contrast with the surrounding brain parenchyma and may be suitable for risk stratification, for prediction of response to standard rituximab- or methotrexate-based immunochemotherapy, and for response assessment.

Diagnosis of CNSL remains one of the major clinical challenges. Because symptoms of CNSL cannot be distinguished from symptoms of other brain-derived tumors, definite diagnosis by histopathologic assessment is necessary. In patients receiving corticosteroids, diagnosis by histopathology is often complicated or virtually impossible because of lymphoma cell apoptosis, which further delays effective treatment and may even require repeat stereotactic biopsy after corticosteroid withdrawal to yield the diagnosis of primary CNSL (45). Particularly, when brain structures not readily accessible for stereotactic biopsy are involved, or in older patient with comorbidities who are at risk for intra- or postoperative mortality, accurate imaging modalities are urgently needed (12). Comparing the efficacy of CXCR4-directed imaging in distinguishing CNSL from glioma is of particular interest and should be addressed in prospective clinical trials.

In addition, evaluation of treatment response by standard imaging modalities such as MRI has limitations in defining complete response in a subgroup of patients (13). Our study revealed that CXCR4directed PET with ${ }^{68} \mathrm{Ga}$-pentixafor was indicative of active disease in all patients. The excellent contrast with the surrounding brain parenchyma, which clearly surpasses PET imaging with ${ }^{18} \mathrm{~F}-\mathrm{FDG}$, may have the potential to result in a refinement of current response criteria provided by the International Primary CNS Lymphoma Collaborative Group, especially when MRI alone is not sufficient to define a complete remission.

Although treatment options for primary CNSL have improved over the last few 
decades, a large proportion of patients eventually relapses after therapy (46). High-dose chemotherapy, with subsequent autologous stem cell transplantation, represents a highly efficient consolidation treatment option for CNSL (47). However, the severe toxicity of this treatment regimen makes careful patient selection necessary. The International Extranodal Lymphoma Study Group identified age, performance status, lactate dehydrogenase serum level, cerebrospinal fluid protein concentration, and involvement of deep brain structures as independent prognostic factors for survival of patients with primary CNSL receiving methotrexate-based chemotherapy. This scoring system, however, does not identify patients benefiting from more intense treatment regimens (48). Because CXCR4-directed PET imaging with ${ }^{68} \mathrm{Ga}-$ pentixafor showed potential for prediciting treatment response in this proof-of-concept study, this novel imaging modality might define patients who could profit from a more intense consolidation therapy followed by autologous stem cell transplantation, thereby reducing the necessity of applying this highly toxic treatment to patients who would not benefit from autologous stem cell transplantation.

Whole-brain radiotherapy has shown high response rates in the treatment of CNSL but was abandoned in first-line therapy because of severe neurotoxicity $(49,50)$. This observation indicates that CNSL is highly susceptible to radiation therapy and that neurotoxicity results mainly from off-target effects of whole-brain radiotherapy. CXCR4-directed tumor cell-targeted endoradiotherapy with pentixather, however-based on the high localized radiation effect-might circumvent neurotoxicity caused by whole-brain radiotherapy. Such treatment has already been safely integrated into hematopoietic stem cell transplantation concepts for myeloma and leukemia (51). First, the incorporation of CXCR4-directed endoradiotherapy into first-line treatment might result in higher response rates. Second, less toxic high-dose chemotherapy protocols in combination with CXCR4-directed endoradiotherapy and autologous stem cell transplantation could be applied in older patients with an adverse prognosis.

\section{CONCLUSION}

This proof-of-concept study demonstrated the feasibility of CXCR4-directed PET imaging with ${ }^{68} \mathrm{Ga}$-pentixafor. Despite the limited sample size and the heterogeneous patient population within this study, CXCR4-directed PET imaging with ${ }^{68} \mathrm{Ga}$ pentixafor displayed a high potential for diagnostic applications and warrants prospective clinical trials for imaging and theranostic applications.

\section{DISCLOSURE}

Ulrich Keller and Andreas Buck serve on the advisory board of PentixaPharm, the company developing licensing of pentixafor and pentixather. Hans-Jürgen Wester is coinventor of pentixafor and pentixather and shareholder of Scintomics, which is a shareholder of PentixaPharm. This work received funding from Deutsche Forschungsgemeinschaft SFB 824 (Markus Schwaiger, Hans-Jürgen Wester, and Ulrich Keller) and SFB 1335 (Ulrich Keller), from Stiftung Charite (Ulrich Keller), and from the German Cancer Consortium (Markus Schwaiger, Hans-Jürgen Wester, and Ulrich Keller). No other potential conflict of interest relevant to this article was reported.

\section{ACKNOWLEDGMENTS}

We thank the hematology/medical oncology and nuclear medicine staff members at the institutions in Munich, Würzburg, and Regensburg for their support.

\section{KEY POINTS}

QUESTION: Is CXCR4-directed PET imaging with ${ }^{68} \mathrm{Ga}$-pentixafor feasible to detect lymphoma lesions of the CNS, and does initial PET uptake correlate with treatment response?

PERTINENT FINDINGS: This retrospective analysis revealed that CXCR4-directed imaging is positive in all active lymphoma lesions, provides excellent contrast properties between lymphoma lesions and healthy brain parenchyma, and correlates well with MRI-determined lymphoma lesions. Moreover, in vivo-determined CXCR4 expression by means of PET significantly correlates with response to treatment.

IMPLICATIONS FOR PATIENT CARE: CXCR4-directed PET imaging with ${ }^{68} \mathrm{Ga}$-pentixafor could not only facilitate diagnostic work-up in patients with CNSL but additionally serve as a biomarker for selecting patients with a dismal prognosis who could profit from more intense treatment options.

\section{REFERENCES}

1. Dolecek TA, Propp JM, Stroup NE, Kruchko C. CBTRUS statistical report: primary brain and central nervous system tumors diagnosed in the United States in 2005-2009. Neuro Oncol. 2012;14(suppl 5):v1-v49.

2. Mendez JS, Ostrom QT, Gittleman H, et al. The elderly left behind: changes in survival trends of primary central nervous system lymphoma over the past 4 decades. Neuro Oncol. 2018;20:687-694.

3. Shiels MS, Pfeiffer RM, Besson C, et al. Trends in primary central nervous system lymphoma incidence and survival in the U.S. Br J Haematol. 2016;174:417-424.

4. Villano JL, Koshy M, Shaikh H, Dolecek TA, McCarthy BJ. Age, gender, and racial differences in incidence and survival in primary CNS lymphoma. $\mathrm{Br} J$ Cancer. 2011;105:1414-1418.

5. Hoffman S, Propp JM, McCarthy BJ. Temporal trends in incidence of primary brain tumors in the United States, 1985-1999. Neuro Oncol. 2006;8:27-37.

6. Boehme V, Zeynalova S, Kloess M, et al. Incidence and risk factors of central nervous system recurrence in aggressive lymphoma: a survey of 1693 patients treated in protocols of the German High-Grade Non-Hodgkin's Lymphoma Study Group (DSHNHL). Ann Oncol. 2007;18:149-157.

7. Deckert M, Engert A, Bruck W, et al. Modern concepts in the biology, diagnosis, differential diagnosis and treatment of primary central nervous system lymphoma. Leukemia. 2011;25:1797-1807.

8. Korfel A, Schlegel U. Diagnosis and treatment of primary CNS lymphoma. Nat Rev Neurol. 2013;9:317-327.

9. Doolittle ND, Abrey LE, Shenkier TN, et al. Brain parenchyma involvement as isolated central nervous system relapse of systemic non-Hodgkin lymphoma: an International Primary CNS Lymphoma Collaborative Group report. Blood. 2008;111: 1085-1093.

10. Dreyling M, Campo E, Hermine O, et al. Newly diagnosed and relapsed mantle cell lymphoma: ESMO clinical practice guidelines. Ann Oncol. 2017;28:iv62-iv71.

11. Louis DN, Perry A, Reifenberger G, et al. The 2016 World Health Organization Classification of Tumors of the Central Nervous System: a summary. Acta Neuropathol (Berl). 2016;131:803-820.

12. Toh CH, Castillo M, Wong AM, et al. Primary cerebral lymphoma and glioblastoma multiforme: differences in diffusion characteristics evaluated with diffusion tensor imaging. AJNR Am J Neuroradiol. 2008;29:471-475.

13. Abrey LE, Batchelor TT, Ferreri AJ, et al. Report of an international workshop to standardize baseline evaluation and response criteria for primary CNS lymphoma. J Clin Oncol. 2005;23:5034-5043.

14. Nabavizadeh SA, Vossough A, Hajmomenian M, Assadsangabi R, Mohan S. Neuroimaging in central nervous system lymphoma. Hematol Oncol Clin North Am. 2016;30:799-821. 
15. Baraniskin A, Deckert M, Schulte-Altedorneburg G, Schlegel U, Schroers R. Current strategies in the diagnosis of diffuse large B-cell lymphoma of the central nervous system. Br J Haematol. 2012;156:421-432.

16. Hoang-Xuan K, Bessell E, Bromberg J, et al. Diagnosis and treatment of primary CNS lymphoma in immunocompetent patients: guidelines from the European Association for Neuro-Oncology. Lancet Oncol. 2015;16:e322-e332.

17. Bergner N, Monsef I, Illerhaus G, Engert A, Skoetz N. Role of chemotherapy additional to high-dose methotrexate for primary central nervous system lymphoma (PCNSL). Cochrane Database Syst Rev. 2012;11:CD009355.

18. Ferreri AJ, Cwynarski K, Pulczynski E, et al. Chemoimmunotherapy with methotrexate, cytarabine, thiotepa, and rituximab (MATRix regimen) in patients with primary CNS lymphoma: results of the first randomisation of the International Extranodal Lymphoma Study Group-32 (IELSG32) phase 2 trial. Lancet Haematol. 2016;3:e217-e227.

19. Ferreri AJ, Reni M, Foppoli M, et al. High-dose cytarabine plus high-dose methotrexate versus high-dose methotrexate alone in patients with primary CNS lymphoma: a randomised phase 2 trial. Lancet. 2009;374:1512-1520.

20. Correa DD, Shi W, Abrey LE, et al. Cognitive functions in primary CNS lymphoma after single or combined modality regimens. Neuro Oncol. 2012;14:101108.

21. Hottinger AF, DeAngelis LM, Yahalom J, Abrey LE. Salvage whole brain radiotherapy for recurrent or refractory primary CNS lymphoma. Neurology. 2007;69:1178-1182.

22. Nguyen PL, Chakravarti A, Finkelstein DM, Hochberg FH, Batchelor TT, Loeffler JS. Results of whole-brain radiation as salvage of methotrexate failure for immunocompetent patients with primary CNS lymphoma. J Clin Oncol. 2005;23:1507-1513.

23. Cojoc M, Peitzsch C, Trautmann F, Polishchuk L, Telegeev GD, Dubrovska A. Emerging targets in cancer management: role of the CXCL12/CXCR4 axis. Onco Targets Ther. 2013;6:1347-1361.

24. Kollet O, Spiegel A, Peled A, et al. Rapid and efficient homing of human $\mathrm{CD} 34(+) \mathrm{CD} 38(-/$ low $) \mathrm{CXCR} 4(+)$ stem and progenitor cells to the bone marrow and spleen of NOD/SCID and NOD/SCID/B2m(null) mice. Blood. 2001;97: 3283-3291.

25. Lapidot T, Sirard C, Vormoor J, et al. A cell initiating human acute myeloid leukaemia after transplantation into SCID mice. Nature. 1994;367:645-648.

26. Nagasawa T. CXC chemokine ligand 12 (CXCL12) and its receptor CXCR4. J Mol Med. 2014;92:433-439.

27. Peled A, Petit I, Kollet O, et al. Dependence of human stem cell engraftment and repopulation of NOD/SCID mice on CXCR4. Science. 1999;283:845-848.

28. Zou YR, Kottmann AH, Kuroda M, Taniuchi I, Littman DR. Function of the chemokine receptor CXCR4 in haematopoiesis and in cerebellar development. Nature. 1998;393:595-599.

29. Spoo AC, Lubbert M, Wierda WG, Burger JA. CXCR4 is a prognostic marker in acute myelogenous leukemia. Blood. 2007;109:786-791.

30. Zhao H, Guo L, Zhao H, Zhao J, Weng H, Zhao B. CXCR4 over-expression and survival in cancer: a system review and meta-analysis. Oncotarget. 2015;6:50225040 .

31. Brunn A, Montesinos-Rongen M, Strack A, et al. Expression pattern and cellular sources of chemokines in primary central nervous system lymphoma. Acta Neuropathol (Berl). 2007;114:271-276.

32. Demmer O, Gourni E, Schumacher U, Kessler H, Wester HJ. PET imaging of CXCR4 receptors in cancer by a new optimized ligand. ChemMedChem. 2011;6: 1789-1791.
33. Gourni E, Demmer O, Schottelius M, et al. PET of CXCR4 expression by a ${ }^{68} \mathrm{Ga}-$ labeled highly specific targeted contrast agent. J Nucl Med. 2011;52:1803-1810.

34. Lapa C, Luckerath K, Kleinlein I, et al. ${ }^{68} \mathrm{Ga}$-pentixafor-PET/CT for imaging of chemokine receptor 4 expression in glioblastoma. Theranostics. 2016;6:428-434.

35. Philipp-Abbrederis K, Herrmann K, Knop S, et al. In vivo molecular imaging of chemokine receptor CXCR4 expression in patients with advanced multiple myeloma. EMBO Mol Med. 2015;7:477-487.

36. Vag T, Gerngross C, Herhaus P, et al. First experience with chemokine receptor CXCR4-targeted PET imaging of patients with solid cancers. J Nucl Med. 2016;57: 741-746.

37. Wester HJ, Keller U, Schottelius M, et al. Disclosing the CXCR4 expression in lymphoproliferative diseases by targeted molecular imaging. Theranostics. 2015;5: 618-630.

38. Habringer S, Lapa C, Herhaus $\mathrm{P}$, et al. Dual targeting of acute leukemia and supporting niche by CXCR4-directed theranostics. Theranostics. 2018;8:369-383.

39. Herrmann K, Schottelius M, Lapa C, et al. First-in-human experience of CXCR4-directed endoradiotherapy with ${ }^{177} \mathrm{Lu}$ - and ${ }^{90}$ Y-labeled pentixather in advanced-stage multiple myeloma with extensive intra- and extramedullary disease. J Nucl Med. 2016;57:248-251.

40. Lapa C, Hanscheid H, Kircher M, et al. Feasibility of CXCR4-directed radioligand therapy in advanced diffuse large B-cell lymphoma. J Nucl Med. 2019;60: 60-64.

41. Haug AR, Cindea-Drimus R, Auernhammer CJ, et al. Neuroendocrine tumor recurrence: diagnosis with ${ }^{68} \mathrm{Ga}$-DOTATATE PET/CT. Radiology. 2014;270:517-525.

42. Martin R, Juttler S, Muller M, Wester HJ. Cationic eluate pretreatment for automated synthesis of $\left[{ }^{68} \mathrm{Ga}\right]$ CPCR4.2. Nucl Med Biol. 2014;41:84-89.

43. Drzezga A, Souvatzoglou M, Eiber M, et al. First clinical experience with integrated whole-body PET/MR: comparison to PET/CT in patients with oncologic diagnoses. J Nucl Med. 2012;53:845-855.

44. Zamagni E, Patriarca F, Nanni C, et al. Prognostic relevance of ${ }^{18} \mathrm{~F}$ FDG PET/CT in newly diagnosed multiple myeloma patients treated with up-front autologous transplantation. Blood. 2011;118:5989-5995.

45. Deckert M, Brunn A, Montesinos-Rongen M, Terreni MR, Ponzoni M. Primary lymphoma of the central nervous system: a diagnostic challenge. Hematol Oncol. 2014;32:57-67.

46. Jahnke K, Thiel E, Martus P, et al. Relapse of primary central nervous system lymphoma: clinical features, outcome and prognostic factors. J Neurooncol. 2006;80: 159-165.

47. Ferreri AJM, Cwynarski K, Pulczynski E, et al. Whole-brain radiotherapy or autologous stem-cell transplantation as consolidation strategies after high-dose methotrexate-based chemoimmunotherapy in patients with primary CNS lymphoma: results of the second randomisation of the International Extranodal Lymphoma Study Group-32 phase 2 trial. Lancet Haematol. 2017;4:e510-e523.

48. Ferreri AJ, Blay JY, Reni M, et al. Prognostic scoring system for primary CNS lymphomas: the International Extranodal Lymphoma Study Group experience. J Clin Oncol. 2003;21:266-272.

49. Omuro AM, Ben-Porat LS, Panageas KS, et al. Delayed neurotoxicity in primary central nervous system lymphoma. Arch Neurol. 2005;62:1595-1600.

50. Gavrilovic IT, Hormigo A, Yahalom J, DeAngelis LM, Abrey LE. Long-term followup of high-dose methotrexate-based therapy with and without whole brain irradiation for newly diagnosed primary CNS lymphoma. J Clin Oncol. 2006;24:4570-4574.

51. Maurer S, Herhaus P, Lippenmeyer R, et al. Side effects of CXC-chemokine receptor 4-directed endoradiotherapy with pentixather before hematopoietic stem cell transplantation. J Nucl Med. 2019;60:1399-1405. 